\title{
Análise situacional do estágio curricular supervisionado nos cursos de graduação em Odontologia no Brasil: uma questão de interpretação
}

\author{
Suzely Adas Saliba Moimaz*; Bruno Wakayama***; Artênio José Isper Garbin**; Cléa Adas Saliba \\ Garbin*; Nemre Adas Saliba*
}
* Professora Titular do Departamento de Odontologia Infantil e Social, Faculdade de Odontologia de Araçatuba, UNESP
** Aluno de Mestrado do Programa de Pós-Graduação em Odontologia Preventiva e Social, Faculdade de Odontologia de Araçatuba, UNESP Professor Doutor Adjunto do Departamento de Odontologia Infantil e Social da Faculdade de Odontologia de Araçatuba, UNESP

Recebido em 30/08/2016. Aprovado em 30/10/2016.

\begin{abstract}
RESUMO
O objetivo neste estudo foi analisar a oferta e a inserção do estágio curricular supervisionado nos cursos de graduação em Odontologia no Brasil. Trata-se de um estudo transversal, descritivo quantitativo. Foram realizadas consultas do número e nomes das instituições que oferecem curso de graduação em Odontologia, pelo sítio eletrônico do Ministério da Educação. As páginas eletrônicas de cada curso foram analisadas, verificando as variáveis: carga horária do curso, regime, carga horária destinada ao estágio curricular, semestre em que é ofertado e a sua nomenclatura. Foram excluídos da amostra aqueles que não apresentaram páginas eletrônicas, sem turmas formadas e os que não disponibilizaram via web o projeto político-pedagógico e/ou estrutura curricular. Para o processamento dos dados e análise estatística foi utilizado o software Epi Info 7.0. Dos 249 cursos de graduação em Odontologia credenciados no Brasil, verificou-se que 241(96,8\%) possuíam páginas eletrônicas ativas, sendo que deste percentual, $158(65,6 \%)$ ofertavam o estágio curricular em suas atividades. Do total de 158 cursos analisados, 89,2\% eram de regime semestral e apenas 38\% atenderam às Diretrizes Curriculares Nacionais, destinando 20\% da carga horária total do curso para estágio. Em relação às nomenclaturas, observaram-se 53 variações, sendo as mais frequentes relacionadas às especialidades odontológicas. Os inícios dos estágios foram mais prevalentes no $5^{\circ}$, $7^{\circ}$ e $8^{\circ}$ semestres. Conclui-se que os estágios curriculares são contemplados na maioria das universidades, com cargas horárias e nomenclaturas diversificadas, entretanto, existe um viés em sua interpretação, pois muitas disciplinas de formação são designadas como atividades de estágio.

Descritores: Educação em Odontologia. Estágios. Odontologia. Escolas de Odontologia.
\end{abstract}




\section{INTRODUÇÃO}

Os primeiros cursos superiores da área da saúde no Brasil foram baseados no modelo de ensino originado pelo relatório do educador Abraham Flexner em 1910, que adotava em sua filosofia a abordagem tecnicista, no qual o indivíduo era assistido fora do seu contexto coletivo e o corpo humano considerado uma máquina divisível, sendo suas partes tratadas separadamente ${ }^{1}$. Esse paradigma foi influente em todas as áreas do conhecimento, bem como a odontológica, que adotava em seu eixo curricular a atenção centrada apenas no plano individual e no curativismo, a fragmentação das disciplinas em micro especialidades e a verticalização do modelo de ensino ${ }^{2,3}$.

Ao considerar que a formação odontológica era baseada apenas na atenção estritamente individual, e que as questões humanas, sociais e de integralização do aluno no contexto sociocultural precisavam ser instituídas para o melhor entendimento dos problemas bucais da comunidade, implantou-se o currículo mínimo em $1982^{4}$. Com essa reformulação curricular e a autonomia das Instituições de Ensino Superior (IES) em estabelecer uma estrutura mais flexível e condizente com a realidade, houve a inclusão do estágio extracurricular e do extramuros a fim de integrar as ações de ensino-serviço, e mostrar ao aluno, as necessidades reais da população ${ }^{5,6}$.

A integração ensino-serviço e a vivência fora da universidade possibilitam ao alunado à compreensão do papel do cirurgião-dentista na construção das ações de saúde, assim como, a importância da sua intervenção sob as carências da população. Dessa forma, a atividade extramural propicia ao acadêmico o entendimento da realidade ao qual foi inserido, estimulando as suas competências profissionais na tomada de decisões, capacitando-os para a atuação no serviço assistencial brasileiro ${ }^{7,8}$.
Dada a importância da incorporação do graduando às diferentes realidades sociais, e considerando que muitas Instituições de Ensino Superior (IES) não resolveram totalmente a questão da formação voltada para a integralidade de atenção à saúde, foram aprovadas as Diretrizes Curriculares Nacionais (DCN), pelo Conselho Nacional de Educação em 2002. Esse documento foi proposto a fim de nortear as IES, na reformulação dos Projetos PolíticoPedagógicos (PPP) para os cursos de Odontologia vigentes até então. A necessidade de adequação dos Projetos Político-Pedagógicos foi pautada na perspectiva de melhor qualificar o futuro profissional, tornando-os aptos a atuarem na realidade a qual se inserem com liderança, aptidão para $\mathrm{o}$ trabalho $\mathrm{em}$ equipe $\mathrm{e}$ competências críticas e generalistas ${ }^{9}$.

Com base nessa premissa e em consonância com a DCN, foram retomadas as discussões sobre a necessidade dos estágios como uma ferramenta de entendimento do contexto social e de aproximação dos principais problemas e demanda da população ${ }^{5}$. O estágio supervisionado foi determinado com uma atividade intrínseca na formação odontológica, sendo desenvolvida sob a supervisão docente, de competência gradual, articulada e com carga horária mínima de $20 \%$ do total do curso de Odontologia ${ }^{9}$. Por recomendação da Associação Brasileira de Ensino Odontológico- ABENO, o estágio curricular supervisionado foi instituído com a incumbência de aproximar o aluno da realidade social e econômica, por meio da interação ensino-serviço/ universidade-sociedade, configurando-os como agentes transformadores ${ }^{10}$.

Ao evidenciar os benefícios da integração academia-serviço para a formação de um profissional da saúde capaz de atender as demandas de saúde do país, com práticas sanitárias efetivas que resultem na melhoria da 
qualificação da atenção aos cidadãos que utilizam o Sistema Único de Saúde ${ }^{11}$, foram instituídas pelo Ministério da Saúde, por meio de uma portaria interministerial, o Programa Nacional de Reorientação da Formação Profissional em Saúde - Pró-Saúde em 2005 ${ }^{12}$, e o Programa de Educação pelo Trabalho para a Saúde - PET Saúde em 2010 ${ }^{13}$. Inicialmente foi criado pela portaria $\mathrm{n}^{\mathrm{o}} 2.101$, em 3 de novembro de 2005, o Pró-saúde para os cursos de graduação em Medicina, Enfermagem e Odontologia. A implementação dessa política educacional governamental foi essencial para a consolidação das DCN na formação profissional, assim como, para a compreensão dos determinantes sociais e da realidade da saúde do país. Posteriormente, com os relatos positivos do programa, como uma ação enriquecedora no processo formador a partir da integração academia-serviço, foi implantando o PET-Saúde em 2010, cujas metas foram semelhantes ao Pró-saúde. Esse programa além de firmar ao que foi postulado, sedimentou a valorização da interdisciplinaridade e o processo de ensino-aprendizagem fora dos muros universitários, por meio dos estágios ${ }^{14,15}$.

Várias discussões estão sendo pautadas sobre as características do "estágio" em decorrência dos vieses de compreensão dos seus fundamentos nos cursos de graduação em Odontologia, seus objetivos, carga horária e vínculo às disciplinas de formação. Com base nisso, o objetivo nessa pesquisa foi analisar a oferta e a inserção do estágio curricular supervisionado nos cursos de Odontologia brasileiros.

\section{MATERIAL E METODOS}

Trata-se de um estudo descritivo quantitativo dos estágios curriculares supervisionados dos cursos de graduação em Odontologia existentes no Brasil.

Foram analisadas as características dos estágios supervisionados descritos nos projetos político-pedagógicos e/ou estruturas curriculares das instituições de ensino superior disponibilizados na internet. Para a coleta de informações sobre o número e nomes das instituições existentes, foi consultada a página eletrônica do Ministério da Educação-MEC http://emec.mec.gov.br/ em agosto de 2015. As instituições que não apresentaram sítios na internet foram excluídas da pesquisa, assim como aquelas sem turmas formadas e as que não disponibilizaram via web o projeto políticopedagógico e/ou estrutura curricular.

Após a obtenção da listagem geral dos cursos de Odontologia do Brasil, iniciaram-se as buscas dos projetos político-pedagógicos e ou estruturas curriculares, nas páginas eletrônicas de cada IES, pela rede mundial de computadores. Nos documentos, analisaram-se as seguintes variáveis: carga horária total do curso, regime, carga horária destinada para a atividade de estágio supervisionado, semestre em que é ofertado e a nomenclatura a essa atividade.

Os estágios foram classificados, de acordo com as suas nomenclaturas em 4 categorias que dimensionam e caracterizam o perfil da atividade curricular executada.

Estágio clínico: determinada pelo público alvo ao qual se destina ou habilidade clínica executada.

Estágio em especialidades odontológicas: relacionadas às especialidades odontológicas realizadas em disciplinas de formação;

\section{Estágio curricular específico no SUS:} atividades conduzidas em conjunto aos serviços públicos de saúde, fora dos muros universitários;

Estágio curricular inespecífico: estágios curriculares sem especificação da localidade da sua execução. Foram incluídos nessa categoria, os chamados "estágios supervisionados" realizados em mais de um semestre, referenciados com algarismos romanos sequenciais. 
Por se tratar de pesquisa com dados secundários públicos dispensa-se a avaliação por comitê de ética.

Para o processamento dos dados, utilizouse o software Epi Info 7.1 for Windows. Na análise dos dados, foi empregada a estatística descritiva, pelas medidas de tendência central e dispersão, representada por meio de tabelas.

\section{RESULTADOS}

Dos 249 cursos de graduação em Odontologia credenciados no MEC, 241(96,8\%) apresentavam sítios eletrônicos disponíveis na web. Desse total, verificou-se que $76,3 \%$ são cursos de instituições privadas, $89,2 \%$ em regime semestral e $76,0 \%$ tem carga horária total do curso variando de 4001 a 5000 horas (tabela 1).

Em relação à carga horária de estágio, 36,7\% variaram de 801 a 1100 horas, 38\% atendiam aos $20 \%$ estipulado pelas DCN e os semestres mais frequentes para o início foram o $5^{\circ}, 7^{\circ}$ e $8^{\circ}$ (tabela 2).

Observou-se que a maioria $(94,3 \%)$ dos cursos de Odontologia que ofertavam o estágio, utilizava nomenclaturas específicas para a atividade. Quanto aos nomes atribuídos aos estágios, os mais comumente empregados foram aqueles vinculados às disciplinas de especialidades formadoras (tabela 3).

Tabela 1. Distribuição percentual dos cursos de Odontologia do Brasil, segundo suas características institucionais, 2015.

\begin{tabular}{lccc}
\hline \multicolumn{1}{c}{ VARIÁVEIS } & & n & \% \\
\hline Cursos & Credenciado & 249 & 98,8 \\
& Descredenciado & 3 & 1,2 \\
& Total & 252 & 100 \\
\hline Sítio eletrônico & Sim & 241 & 96,8 \\
& Não & 8 & 3,2 \\
& Total & 249 & 100 \\
\hline Categoria & Público & 57 & 23,7 \\
& Particular & 184 & 76,3 \\
& Total & 241 & 100 \\
\hline Regime & Anual & 26 & 10,8 \\
& Semestral & 215 & 89,2 \\
& Total & 241 & 100 \\
\hline Período & Integral & 228 & 94,6 \\
& Noturno & 13 & 5,4 \\
& Total & 241 & 100 \\
\hline Carga Horária & Até 4000 & 35 & 14,5 \\
& 4001 a 5000 & 183 & 76,0 \\
& 5001 a 5955 & 23 & 9,5 \\
& Total & 241 & 100 \\
\hline Projeto Pedagógico & Sim & 31 & 12,9 \\
& Não & 210 & 87,1 \\
& Total & 241 & 100 \\
\hline Estrutura Curricular & Sim & 199 & 82,6 \\
& Não & 42 & 17,4 \\
& Total & 241 & 100 \\
\hline Estágio Curricular & Sim & 158 & 65,6 \\
& Não & 83 & 34,4 \\
& Total & 241 & 100 \\
\hline
\end{tabular}


Tabela 2. Distribuição numérica e percentual dos estágios, segundo as variáveis do estudo, 2015.

\begin{tabular}{cccc}
\hline VARIÁ VEIS & n & \% \\
\hline Nomenclatura dos estágios & Não especificado & 9 & 5,7 \\
& Especificado & 149 & 94,3 \\
& Indisponível & 30 & 19,0 \\
Carga Horária & Total & 158 & 100 \\
\hline Até 500 & 28 & 17,7 \\
& 501 a 800 & 32 & 23,3 \\
& 801 a 1100 & 58 & 36,7 \\
& 1101 a 1400 & 4 & 2,5 \\
& $>1401$ & 6 & 3,8 \\
& Total & 158 & 100 \\
\hline Semestre do início do Estágio & Indisponível & 30 & 19,0 \\
& Sim & 60 & 38,0 \\
& Não & 68 & 43,0 \\
& Total & 158 & 100 \\
\hline Indisponível & 1 & 27 & 17,1 \\
& 2 & 11 & 7,0 \\
& 3 & 11 & 7,0 \\
& 4 & 10 & 6,3 \\
& 5 & 15 & 9,5 \\
& 6 & 18 & 11,4 \\
& 7 & 16 & 10,1 \\
& 8 & 18 & 11,4 \\
& 9 & 19 & 12,0 \\
& 10 & 10 & 6,3 \\
& 11 & 2 & 1,3 \\
& 12 & 0 & 0 \\
& Total & 158 & 0,6 \\
& & & 100 \\
\hline
\end{tabular}

\section{DISCUSSÃO}

Nesse estudo sobre os estágios curriculares nos cursos de Odontologia no Brasil, mais da metade dos cursos analisados apresentavam o estágio curricular em suas atividades, entretanto, apenas $38 \%$ estavam de acordo com a carga horária estipulada pelas DCN.

Na década de 80, os estágios curriculares supervisionados, eram desenvolvidos por meio das atividades extramuros, orientados pela Resolução CFE n ${ }^{\circ}$ 04/82 do Conselho Federal de Educação, que estabeleceu o chamado currículo mínimo para as IES. Neste documento, pautavam-se as atividades a serem executadas como estágio, a inserção do aluno fora dos muros da universidade em contato direto com o contexto social do país, a fim de formar profissionais hábeis para aplicar os princípios biológicos, técnicos e éticos para resolução das doenças bucais mais prevalentes na população ${ }^{4}$.

Naquele momento, houve uma grande evolução nas implementações dos estágios extramuros nas universidades, contudo, ainda se observavam dificuldades no atendimento dos objetivos e habilidades, inicialmente propostos pelos estágios supervisionados.

Assim sendo, no início dos anos 2000, com o surgimento das DCN, consolidaram-se ações 
Tabela 3. Classificação dos "estágios" de acordo com as nomenclaturas adotadas, 2015

\begin{tabular}{|c|c|c|}
\hline Estágio clínico & $\mathbf{n}$ & $\%$ \\
\hline Acompanhamento clínico & 5 & 3,16 \\
\hline Estágio clínico & 2 & 1,27 \\
\hline Clínica do adulto & 3 & 1,90 \\
\hline Clínica da criança e do adolescente & 1 & 0,63 \\
\hline Odontologia geral & 2 & 1,27 \\
\hline Clínica do idoso & 2 & 1,27 \\
\hline Clínica de propedêutica odontológica & 1 & 0,63 \\
\hline Clínica infantil & 10 & 6,32 \\
\hline Clínica odontológica & 2 & 1,27 \\
\hline \multicolumn{3}{|l|}{ Estágio por Especialidades } \\
\hline Anestesiologia & 1 & 0,63 \\
\hline Cirurgia & 9 & 5,70 \\
\hline Dentística & 6 & 3,80 \\
\hline Diagnóstico & 2 & 1,27 \\
\hline DTM & 1 & 0,63 \\
\hline Urgência e emergência & 23 & 14,56 \\
\hline Endodontia & 4 & 2,53 \\
\hline Estomatologia & 8 & 5,06 \\
\hline Odontogeriatria & 5 & 3,16 \\
\hline Odontologia do bebê & 1 & 0,63 \\
\hline Odontologia hospitalar & 8 & 5,06 \\
\hline Odontopediatria & 39 & 24,69 \\
\hline Odontologia preventiva e social & 3 & 1,90 \\
\hline Ortodontia & 1 & 0,63 \\
\hline Pacientes especiais & 11 & 6,96 \\
\hline Periodontia & 3 & 1,90 \\
\hline Prótese & 12 & 7,59 \\
\hline Reabilitação & 3 & 1,90 \\
\hline Odontologia restauradora & 1 & 0,63 \\
\hline Saúde coletiva & 34 & 21,52 \\
\hline Clínica integrada & 70 & 44,30 \\
\hline Estágio específico no SUS & $\mathbf{n}$ & $\%$ \\
\hline Extramuro & 24 & 15,18 \\
\hline $\mathrm{ESF}^{*}$ e APS** & 12 & 7,59 \\
\hline Atenção básica e atenção integral & 4 & 2,53 \\
\hline Centro comunitário & 3 & 1,90 \\
\hline Estágio no SUS & 12 & 7,59 \\
\hline CRUTAC $* * *$ & 1 & 0,63 \\
\hline Estágio na Unidade Básica de saúde & 4 & 2,53 \\
\hline Gestão de saúde pública & 1 & 0,63 \\
\hline Sistema público de saúde & 5 & 3,16 \\
\hline Serviços de saúde & 4 & 2,53 \\
\hline Saúde pública & 3 & 1,90 \\
\hline \multicolumn{3}{|l|}{ Estágio curricular inespecífico } \\
\hline Internato & 1 & 0,63 \\
\hline Multidisciplinar e interdisciplinar & 3 & 1,90 \\
\hline Estágio supervisionado & 53 & 33,54 \\
\hline Estágio em alta complexidade e hospitalar & 6 & 3,80 \\
\hline Promoção de saúde bucal & 1 & 0,63 \\
\hline Educação em saúde & 2 & 1,27 \\
\hline Práticas epidemiológicas & 2 & 1,27 \\
\hline Estágio vivencial & 2 & 1,27 \\
\hline Triagem & 3 & 1,90 \\
\hline Informática e documentação & 2 & 1,27 \\
\hline Laboratorial & 1 & 0,63 \\
\hline Estágio profissional & 1 & 0,63 \\
\hline
\end{tabular}


norteadoras que estimulassem as universidades a reformularem seus projetos político-pedagógicos para formação de profissionais mais críticos e generalistas e foram estabelecidos critérios restritos para a atividade dos estágios curriculares supervisionados ${ }^{16}$.

As atividades desenvolvidas nos estágios, ainda se configuram como uma questão controversa gerando discussões sobre "onde", "como", e "quando" devem ser realizados, dentre outras competências. Isto é decorrente, do seu viés de interpretação, assim como, pela atribuição genérica que é estabelecida a essa atividade, que muitas vezes, é fundamentada e conduzida sem critérios e vinculada às disciplinas de especialidade formadora. Dada essa dimensão, em 2015 foi realizada pela ABENO, a revisão das Diretrizes Curriculares Nacionais para a definição do Estágio Supervisionado Curricular, a fim de nortear e esclarecer alguns aspectos a eles relacionados ${ }^{17}$.

O estágio curricular supervisionado tem o compromisso de explorar as habilidades do acadêmico como um instrumento de aprimoramento técnico-científico, no qual as vivências em vários cenários e contextos sociais lhes proporcionem o senso crítico, responsável e consciente sobre a importância da promoção de saúde e do atendimento integral e humanizado ${ }^{18}$. O desenvolvimento dessas habilidades e competências são características essenciais para a formação de um profissional capaz de atender e suprir às demandas do sistema público de saúde do Brasil. A Constituição Federal no seu artigo 200, inciso III determina a competência do Sistema Único de Saúde - SUS em, "ordenar a formação de recursos humanos na área de saúde" ${ }^{19}$. Além disso, a legislação brasileira que regulamenta as ações e serviços de saúde no território brasileiro - lei 8080, reitera a importância de profissionais para o serviço público, ao estabelecer no artigo 6 , inciso III, " $a$ ordenação da formação de recursos humanos na área de saúde"; e pelo artigo 15, inciso IX, "participação na formulação e na execução da política de formação e desenvolvimento de recursos humanos para a saúde"20.

Estudos internacionais têm evidenciado grandes benefícios na formação do futuro egresso em Odontologia, ao se inserirem nos contextos sociais fora das instalações da universidade, e depará-los com a carência em saúde bucal de grande parte da população sem acesso à atenção privada em saúde. No estudo realizado em uma universidade norte americana, os pesquisadores ressaltaram que a vivência dos alunos com os problemas bucais reais da comunidade a qual estão inseridos, gera reflexões sobre a problemática enfrentada e a conscientização das diversidades culturais e étnicas ${ }^{21}$. Coe et al. ${ }^{22}$ ao empregar uma metodologia para analisar como o programa de ensino-serviço realizado na Virginia-EUA interfere na atitude dos acadêmicos para atenção a saúde bucal, concluíram que as atividades executadas tiveram impactos positivos em algumas dimensões, gerando benefícios para os participantes envolvidos. Pesquisas nacionais enfatizam as experiências e percepções dos graduandos sobre as atividades extramurais, e ressaltam a valorização dessa atividade para o comprometimento e engajamento dos discentes com as práticas de ensino, sendo o contato direto com a realidade e o contexto social, a principal característica de agregação de experiência profissional $^{5,7,23,24}$.

Verificou-se nessa pesquisa, que grande parte dos cursos de graduação em Odontologia tinham a carga horária destinada aos estágios supervisionados, inferiores aos 20\% da carga horária total do curso. Scavuzzi et al. ${ }^{17}$ discutem que a atividade do estágio curricular deve corresponder a $20 \%$ da carga horária total do curso. Além disso, é considerada condição ideal, 
o cumprimento de $100 \%$ da carga horária destinada aos estágios em atividades nos cenários do SUS. Entretanto, conforme discutido neste mesmo documento, a distribuição da carga horária torna-se suficiente, quando destinado $50 \%$ para a condução do estágio em cenários extramuros, sendo que, obrigatoriamente, no mínimo $25 \%$ deverá corresponder às atividades destinadas ao SUS.

Existem várias possibilidades da realização de estágios no sistema público de saúde, fora dos muros da universidade, em todos os níveis operacionais. Essas atividades podem ser desenvolvidas na Atenção Primária: nas Unidades Básicas de Saúde (UBS) e Estratégia da Saúde da Família (ESF), realizando ações e acompanhando o trabalho da Equipe de Saúde Bucal; na Atenção Secundária em Centros de Especialidades Odontológicas; na Atenção Terciária em hospitais e centros de referências de serviços especializados. Além disso, os estágios também podem ser realizados em órgãos que compõem o SUS como a Vigilância sanitária, Vigilância epidemiológica, Vigilância ambiental e da Vigilância em saúde do trabalhador, na área de gestão e planejamento das Secretarias da Saúde dentre outras.

Fonseca et al. ${ }^{25}$ ao construir um modelo lógico-ideal para a condução dos estágios curriculares supervisionados, ressaltaram a relevância de incluir o alunado em todas as esferas de organização dos serviços de saúde. Essa experiência colaborará para a maior compreensão do processo de atenção e serviços oferecidos pelo SUS, apropriando-o da responsabilização e desmistificando-o de préconceitos. Dessa maneira, o início e a distribuição dos estágios na estrutura curricular, devem ser baseados no nível de desenvolvimento das habilidades e competências dos alunos, sendo incluídas de forma longitudinal e de complexidade crescente ao longo da atividade acadêmica.

Em relação às condições que determinam as atividades de estágios curriculares, conforme estabelecido pela DCN e discutido pela ABENO, não são consideradas como estágio, situações que envolvem o atendimento de pacientes para o desenvolvimento de uma habilidade técnica e ou uma prática clínica, como exemplificado por Scavuzzi et al. "As clínicas isoladas de uma, duas ou três especialidades e aulas práticas laboratoriais não são consideradas para efeito de estágio supervisionado"17.

A diversidade de nomes atribuídos às atividades de "estágios" demonstram a complexidade do tema, e a importância do esclarecer a sua interpretação. Outros estudos devem ser realizados na forma de inquérito com gestores e docentes das disciplinas, para que haja consenso sobre os objetivos dessa atividade. Dessa forma, torna-se necessário o debate com educadores e organizações ligadas ao ensino Odontológico, a fim de esclarecer e elucidar os achados sobre os estágios curriculares observados nessa pesquisa.

\section{CONCLUSÃO}

Conclui-se que os estágios curriculares são contemplados na grande maioria dos cursos de Odontologia, com cargas horárias e nomenclaturas diversificadas, entretanto, há diferentes formas de compreensão, pois suas características não estão totalmente de acordo com as Diretrizes Curriculares Nacionais e muitas disciplinas de formação são ainda designadas como atividades de estágio.

\section{ABSTRACT \\ Situational analysis of supervised curricular internship in Brazilian Dental Schools: a matter of interpretation}

The goal of this study was to verify the offer and insertion of supervised curricular internship in undergraduate dental schools in Brazil. This was a transversal descriptive quantitative study. The 
number and names of institutions that offer undergraduate courses in dentistry were searched at the Ministry of Education website. Every faculty's website was analyzed, and the following variables were verified: workload, regimen, internship workload, semester in which it is offered and nomenclature. The ones that either had no electronic home pages, or had not graduated any students, or displayed no political pedagogical project and/or the course curriculum via web were left out of the sample. Epi Info 7.0 software was used for data processing and statistical analysis. Out of 249 undergraduate dental schools catalogued in Brazil, it was observed that $241(96.8 \%)$ had active home pages, among which 158 (65.6\%) offered curricular internship among their activities. Of the 158 analyzed courses, only $38 \%$ responded to the National Curricular Parameters (NCPs), assigning $20 \%$ of the workload to internship, and $89.2 \%$ were semester-oriented. Regarding nomenclature, 53 variations were observed, the most frequent related to dental specialties. The beginning of the internships was most prevalent in the $5^{\text {th }}, 7^{\text {th }}$ and $8^{\text {th }}$ semesters. It can be concluded that curricular internships are comprehended in most faculties, with different workloads and nomenclature; however, this nomenclature may be biased, since several technical dental courses are designated as internship activities.

Descriptors: Education Dental. Internships. Dentistry. Dental Schools.

\section{REFERÊNCIAS}

1. Flexner A. Medical education in the United States and Canada. New York: Carnegie Foundation for The Advancement of Teaching; 1910.

2. Pelissari LD, Basting RT, Florio FM. Vivência da realidade: o rumo da saúde para a Odontologia. Rev ABENO. 2005;5(1):329.

3. Toassi RFC, Stobäus CD, Mosquera JJM, Moysés SJ. Currículo integrado no ensino de Odontologia: novos sentidos para a formação na área da saúde. Interface Comun Saúde Educ. 2012;16(41):529-42.
4. Brasil. Parecer n ${ }^{\circ}$ 370, de 9 de julho de 1982. Proposta do novo currículo mínimo para o Curso de Graduação em Odontologia CCC. Documenta. 1982;260:46-54.

5. Leme PAT, Pereira AC, Meneghim MC, Mialhe FL. Perspectivas de graduandos em odontologia acerca das experiências na atenção básica para sua formação em saúde. Ciênc Saúde Coletiva. 2015;20(4):1255-65.

6. Moimaz SAS, Saliba NA, Garbin CAS, Zina LG, Furtado JF, Amorim JA. Serviço extramuro odontológico: impacto na formação profissional. Pesqui Bras Odontopediatria Clin Integr. 2004;4(1):53-7.

7. Moimaz SAS, Saliba NA, Garbin CAS, Zina LG. Atividades extramuros na ótica de egressos do curso de graduação em odontologia. Rev ABENO. 2008;8(1):23-9.

8. Segura MEC, Soares MS, Jorge WA. Programas extramuros nas instituições de ensino de odontologia na América Latina e nos Estados Unidos da América: contribuição ao estudo. Educ Méd Salud. 1995;29(2):218-27.

9. Brasil. Conselho Nacional de Educação. Câmara de Educação Superior. Resolução CNE/CES 3, de 19 de fevereiro de 2002. Institui Diretrizes Curriculares Nacionais do Curso de Graduação em Odontologia. Diário Oficial da União 2002; 4 mar. [Acesso em 03 ago. 2016]. Disponível em: http://portal. mec.gov.br/cne/arquivos/pdf/CES032002.pdf

10. Associação Brasileira de Ensino Odontológico. Diretrizes da ABENO para a definição do estágio supervisionado nos cursos de Odontologia. Rev ABENO. 2002;2(1):39.

11. Silva MAM, Amaral JHL, Senna MIB, Ferreira EF. The national reorientation of vocational healthcare training (Pro-Saúde) and encouragement of inclusion of differentiated learning scenarios within dentistry courses in Brazil. Interface Comun Saúde Educ. 2012;16(42):707-17.

12. Brasil. Ministério da Saúde. Ministério da Educação. Portaria interministerial $\mathrm{n}^{\circ} 2.101$, de 3 de novembro de 2005. Institui o Programa Nacional de Reorientação da 
Formação Profissional em Saúde - PróSaúde - para os cursos de Graduação em Medicina, Enfermagem e Odontologia. Diário Oficial da União 2005; nov. 4. [Acesso em 03 ago. 2016]. Disponível em: http://www.portaleducacao.com.br/odontol ogia/artigos/3454/portaria-interministerialms-mec-n-2101-de-3-de-novembro-de-2005

13. Brasil. Ministério da Saúde. Ministério da Educação. Portaria Interministerial $n^{\circ} 422$, de 3 de março de 2010. Estabelece orientações e diretrizes técnicoadministrativas para a execução do Programa de Educação pelo Trabalho para a Saúde - PET Saúde, instituído no âmbito do Ministério da Saúde e do Ministério da Educação. Diário Oficial da União 2010; mar 5. [Acesso em 03 ago. 2016]. Disponível em: http://bvsms.saude.gov.br/ bvs/saudelegis/gm/2010/pri0422 $03 \quad 03 \quad 20$ 10.html

14. Ferreira JR, Haddad AE, Brenelli SL, Cury GC, Puccini RF, Martins MA, Campos FE. Pró-Saúde e PET-Saúde: experiências exitosas de integração ensino-serviço. Rev Bras Educ Méd. 2012;36(1):3-4.

15. Souza GCLL, Lopes MRL, Rodrigues CAQ, Moreira KS, Oliveira MP, Morais AJD, Leite MTS. O PET- Saúde na formação acadêmica em odontologia: contribuições e perspectivas. Rev ABENO. 2014;14(1):7380.

16. Moimaz SAS, Fadel CB, Bino LS, Saliba NA. Projeto pedagógico e estrutura curricular de um curso de odontologia: análise crítica fundamentada na percepção acadêmica. Rev ABENO. 2010;10(2):35-40.

17. Scavuzzi AIF, Gouveia CVD, Carcereri DL, Veeck EB, Ranali J, Costa LJ, Morita MC, Araújo ME. Revisão das Diretrizes da ABENO para a definição do estágio supervisionado curricular nos cursos de Odontologia. Rev ABENO. 2015;15(3):10913.

18. Francisco KMS, Moimaz SAS, Diniz DG, Saliba NA. Oferta e distribuição de estágio supervisionado em cursos de odontologia do estado de Minas Gerais, Brasil. Biosci J.
2010;26(1):152-60.

19. Brasil. Constituição da República Federativa do Brasil. 35th ed. São Paulo: Biblioteca Digital Câmara; 2012.

20. Brasil. Lei $\mathrm{n}^{\circ} 8080$ de 19 de setembro de 1990. Dispõe sobre as condições para a promoção, proteção e recuperação da saúde, a organização e o funcionamento dos serviços correspondentes e dá outras providências. Diário Oficial da União 1990; set 20. [Acesso em 03 ago. 2016]. Disponível em: http://conselho.saude.gov.br/legislacao/ lei8080 190990.htm

21. Aston-Brown RE, Branson B, GadburyAmyot CC, Bray KK. Utilizing public health clinics for service-learning rotations in dental hygiene: a four-year retrospective study. J Dent Educ. 2009;73(3):358-74.

22. Coe JM, Best AM, Warren JJ, McQuistan MR, Kolker J, Isringhausen KT. Servicelearning's impact on dental students' attitude towards community service. Eur J Dent Educ. 2015;19(3):131-9.

23. Silva BS, Caldarelli PG. O PET-Saúde em consonância com as Diretrizes Curriculares Nacionais de Odontologia no desenvolvimento de competências profissionais: relato de experiência. Rev ABENO. 2013; 13(2):34-41.

24. Santos KT, Ferreira L, Batista RJ, Bitencourt CTF, Araujo RP, Carvalho RB. Percepção discente sobre a influência de estágio extramuro na formação acadêmica odontológica. Rev Odontol UNESP. 2013; 42(6):420-5.

25. Fonseca GS, Junqueira SR, Araújo ME, Botazzo C. Modelo lógico-ideal para o estágio curricular supervisionado: a educação pelo trabalho na formação Odontológica. Rev ABENO. 2015;15(2):2-11.

Correspondência para:

Profa. Dra. Suzely Adas Saliba Moimaz

e-mail: sasaliba@foa.unesp.br

Programa de Pós-Graduação em Odontologia

Preventiva e Social

Rua José Bonifácio, 1193 - Vila Mendonça 16015-050 Araçatuba/SP 